\title{
ORTHOGONAL MULTI-WAVELETS FROM MATRIX FACTORIZATION
}

\author{
HONGYing XiAO
}

\begin{abstract}
Accuracy of the scaling function is very crucial in wavelet theory, or correspondingly, in the study of wavelet filter banks. We are mainly interested in vector-valued filter banks having matrix factorization and indicate how to choose block central symmetric matrices to construct multi-wavelets with suitable accuracy.
\end{abstract}

\section{Introduction}

We consider the case of compactly supported multi-wavelets. That is, suppose $\Phi=\left(\phi_{1}, \ldots, \phi_{r}\right)^{T}$ are scaling functions, and $\Psi=\left(\psi_{1}, \ldots, \psi_{r}\right)^{T}$ are the corresponding wavelet functions, so that the following two-scale equations hold for all $x \in \mathbb{R}$ :

$$
\begin{aligned}
& \Phi(x)=\sum_{n=0}^{M} S_{n} \Phi(2 x-n), \\
& \Psi(x)=\sum_{n=0}^{M} T_{n} \Phi(2 x-n) .
\end{aligned}
$$

Define the corresponding symbol functions as

$$
m_{0}(x):=\frac{1}{2} \sum_{n \in \mathbb{Z}} S_{n} x^{n}, \quad m_{1}(x):=\frac{1}{2} \sum_{n \in \mathbb{Z}} T_{n} x^{n} .
$$

As is well known, the orthonormality of the multi-wavelets implies the following PR condition

$$
H(x) H^{T}(1 / x)=I_{2},
$$

with

$$
H(x):=\left(\begin{array}{cc}
m_{0}(x) & m_{0}(-x) \\
m_{1}(x) & m_{1}(-x)
\end{array}\right)
$$

Received June 6, 2007; Revised June 3, 2008.

2000 Mathematics Subject Classification. 42C40, 65T60.

Key words and phrases. multi-wavelets, PR condition, accuracy, block central symmetric matrix.

(C)2009 The Korean Mathematical Society 
Up to now, it seems very difficult to find all solutions of the matrix equation (1.3) so people hope to construct some special solutions. A class of PR wavelet filter banks was given in [2] for the general case. In particular, one solution for the multi-wavelets case is as follows:

$$
m_{k}(x)=\frac{1}{2}\left(I_{r}, x I_{r}\right)\left[\prod_{j=1}^{N} U_{j} \operatorname{diag}\left(I_{r}, x^{2} I_{r}\right) U_{j}^{T}\right] V_{k}, k=0,1 .
$$

Here $N$ is a fixed positive integer, $U_{j}$ is any arbitrary $2 r \times 2 r$ real orthogonal matrix, and

$$
V:=\left(V_{0}, V_{1}\right)=\left(\begin{array}{cc}
I_{r} & I_{r} \\
I_{r} & -I_{r}
\end{array}\right)
$$

The following theorem could be found in [2].

Theorem 1.1. Suppose filter banks are constructed as in (1.5)-(1.6). Then the PR condition (1.3) is satisfied.

It is well known that the linear phase of filter banks corresponds to symmetry of the related functions. It was also pointed out in [2] that to ensure the uniform linear phase, i.e., to ensure that there exists a natural number $s$ such that $m_{k}(x)=x^{s} m_{k}(1 / x), k=0,1$, we should choose $U_{j}$ to be $r$-block central symmetric matrices:

$$
U_{j}=S\left(\begin{array}{cc}
P_{j} & 0 \\
0 & Q_{j}
\end{array}\right) S^{T}, S=\left(\begin{array}{cc}
I_{r} & -J_{r} \\
J_{r} & I_{r}
\end{array}\right),
$$

where $P_{j}, Q_{j}$ are $r$-th real orthogonal matrices and $J_{r}$ is the $r$-th reversal matrix.

For later convenience, let $G(x)=\frac{1}{2}\left(I_{r}, x I_{r}\right) \prod_{j=1}^{N} U_{j} \operatorname{diag}\left(I_{r}, x^{2} I_{r}\right) U_{j}^{T}$.

\section{Accuracy conditions of multiple scaling functions}

In the following sections, we concentrate on sufficient and necessary conditions so that the scaling functions have accuracy of order $p$. That is, all polynomials with total degree at most $p-1$ can be reproduced from linear combinations of the multi-integer translates of function $\Phi$.

Gilbert Strang stated in [5] that to ensure accuracy, one must check the value of function $m_{0}$ and its derivatives at all aliasing frequencies which seems difficult to compute. By only imposing some conditions on the functions $m_{0}, m_{1}$ at $x=1$, the author of this paper produced the following theorem, [9].

Theorem 2.1. If $\Phi=\left(\phi_{1}, \phi_{2}, \ldots, \phi_{r}\right)^{T}$ are scaling functions, and the integer translates of $\phi_{1}, \ldots, \phi_{r}$ are linearly independent, moreover, if the corresponding filter bank satisfies the PR condition (1.3), then $\Phi$ have accuracy $p$ if and only 
if there are $p$ vectors $\nu_{0}, \ldots, \nu_{p-1}$, each $\nu_{l}$ being $r \times 1$ vector and $\nu_{0} \neq 0$, such that for all $j \in \mathbb{Z}_{p}=\{0,1, \ldots, p-1\}$ :

$$
\begin{gathered}
\sum_{l=0}^{j}\left(\begin{array}{l}
j \\
l
\end{array}\right)(2 i)^{l-j} m_{0}^{(j-l)}(1) \nu_{l}=2^{j} \nu_{j}, \\
\sum_{l=0}^{j}\left(\begin{array}{l}
j \\
l
\end{array}\right)(2 i)^{l-j} m_{1}^{(j-l)}(1) \nu_{l}=0 .
\end{gathered}
$$

By using this theorem, the next proposition is obtained for the filter banks constructed as above.

Proposition 2.2. If $\Phi=\left(\phi_{1}, \phi_{2}, \ldots, \phi_{r}\right)^{T}$ are scaling functions with the integer translates of $\phi_{1}, \ldots, \phi_{r}$ being linearly independent, and, the corresponding filter banks are constructed as in (1.5)-(1.6), then $\Phi$ have accuracy $p$ if and only if there are $p$ vectors $\nu_{0}, \ldots, \nu_{p-1}$, each $\nu_{l}$ being $r \times 1$ vector and $\nu_{0} \neq 0$, such that for all $j \in \mathbb{Z}_{p}$ :

$$
\begin{gathered}
\sum_{l=0}^{j}\left(\begin{array}{l}
j \\
l
\end{array}\right)(2 i)^{l-j} G^{(j-l)}(1) V_{0} \nu_{l}=2^{j} \nu_{j}, \\
\sum_{l=0}^{j}\left(\begin{array}{l}
j \\
l
\end{array}\right)(2 i)^{l-j} G^{(j-l)}(1) V_{1} \nu_{l}=0 .
\end{gathered}
$$

By this proposition, we must find $p$ vectors $\nu_{0}, \ldots, \nu_{p-1}$ to meet the requirements of equations (2.3) and (2.4). The procedure is simplified as follows so that one must only find a nonzero vector $\nu_{0}$ which is the common eigen-vector corresponding to eigenvalue $\lambda=0$ of several matrices.

Theorem 2.3. Under the assumptions of Proposition 2.2, the scaling functions have accuracy $p$ if and only if there exist a $r \times 1$ vector $\nu_{0} \neq 0$ such that $\nu_{0}$ are common eigenvector corresponding to eigenvalue 0 of matrices $B_{1}, \ldots, B_{p-1}$, that is,

$$
B_{n} \nu_{0}=0, n=1,2, \ldots, p-1 .
$$

The matrices $B_{j}$ are constructed iteratively as

$$
\left\{\begin{array}{l}
B_{1}=N_{1}, \\
B_{n}=N_{n}+\sum_{j=1}^{n-1} \frac{\left(\begin{array}{c}
n \\
j
\end{array}\right)}{2^{j}-1} N_{n-j} A_{j}, 2 \leq n \leq p-1
\end{array}\right.
$$

and

$$
\left\{\begin{array}{l}
A_{1}=M_{1}, \\
A_{n}=M_{n}+\sum_{j=1}^{n-1} \frac{\left(\begin{array}{c}
n \\
j
\end{array}\right)}{2^{j}-1} M_{n-j} A_{j}, 2 \leq n \leq p-1
\end{array}\right.
$$


with

$$
M_{j}:=G^{(j)}(1) V_{0}, N_{j}:=G^{(j)}(1) V_{1} .
$$

Furthermore, the solutions of equations (2.3), (2.4) are given as

$$
\nu_{n}=\frac{(2 i)^{-n}}{2^{n}-1} A_{n} \nu_{0}, n=1,2, \ldots, p-1 .
$$

Proof. If the assumptions of Proposition 2.2 are satisfied, one easily checks that

$$
G(1) V_{0}=I_{r}, G(1) V_{1}=0_{r},
$$

so that for any $r \times 1$ vector $\nu_{0} \neq 0$, equations (2.3) and (2.4) for $p=1$. Thus, the corresponding scaling functions have at least accuracy of $p=1$.

From Proposition 2.2, $\Phi$ have accuracy $p=2$ if and only if there exists $r \times 1$ vector $\nu_{0}, \nu_{1}$ with $\nu_{0} \neq 0$ such that

$$
\begin{array}{ll}
G(1) V_{0} \nu_{0}=\nu_{0}, & G(1) V_{1} \nu_{0}=0, \\
(2 i)^{-1} G^{(1)}(1) V_{0} \nu_{0}+G(1) V_{0} \nu_{1}=2 \nu_{1}, & (2 i)^{-1} G^{(1)}(1) V_{1} \nu_{0}+G(1) V_{1} \nu_{1}=0 .
\end{array}
$$

By using the relations (2.10) and the notations in (2.6)-(2.8), the last equations are equivalent to

$$
B_{1} \nu_{0}=0, \nu_{1}=(2 i)^{-1} A_{1} \nu_{0},
$$

this is just what equations (2.5) and (2.9) states for $p=2$.

Suppose this theorem holds for some $p \geq 2$, next we will prove by induction that it also holds for $p+1$. Proposition 2.2 states that $\Phi$ have accuracy $p+1$ if and only if there exists $r \times 1$ vector $\nu_{0}, \ldots, \nu_{p+1}$ with $\nu_{0} \neq 0$ such that $(2.3)$ and (2.4) holds for all $j=0,1, \ldots, p$. By induction, this is equivalent to

$$
\begin{aligned}
& B_{n} \nu_{0}=0, \nu_{n}=\frac{(2 i)^{-n}}{2^{n}-1} A_{n} \nu_{0}, n=1,2, \ldots, p-1 ; \\
& \sum_{l=0}^{p}\left(\begin{array}{l}
p \\
l
\end{array}\right)(2 i)^{l-p} G^{(p-l)}(1) V_{0} \nu_{l}=2^{p} \nu_{p} ; \\
& \sum_{l=0}^{p}\left(\begin{array}{l}
p \\
l
\end{array}\right)(2 i)^{l-p} G^{(p-l)}(1) V_{1} \nu_{l}=0 .
\end{aligned}
$$

By using (2.10) and (2.12), the left side of equation (2.14) equals to

$$
\begin{aligned}
& \sum_{l=1}^{p}\left(\begin{array}{l}
p \\
l
\end{array}\right)(2 i)^{l-p} N_{p-l} \nu_{l}+(2 i)^{-p} N_{p} \nu_{0} \\
= & \sum_{l=1}^{p}\left(\begin{array}{l}
p \\
l
\end{array}\right)(2 i)^{l-p} N_{p-l} \frac{(2 i)^{-l}}{2^{l}-1} A_{l} \nu_{0}+(2 i)^{-p} N_{p} \nu_{0} \\
= & (2 i)^{-p}\left\{\sum_{l=1}^{p} \frac{\left(\begin{array}{l}
p \\
l
\end{array}\right)}{2^{l}-1} N_{p-l} A_{l}+N_{p}\right\} \nu_{0}=(2 i)^{-p} B_{p} \nu_{0} .
\end{aligned}
$$


Similarly, by using (2.10) and (2.12), the left side of equations (2.13) equals to

$$
\begin{aligned}
& \sum_{l=1}^{p}\left(\begin{array}{l}
p \\
l
\end{array}\right)(2 i)^{l-p} M_{p-l} \nu_{l}+(2 i)^{-p} M_{p} \nu_{0}+\nu_{p} \\
= & \sum_{l=1}^{p}\left(\begin{array}{l}
p \\
l
\end{array}\right)(2 i)^{l-p} M_{p-l} \frac{(2 i)^{-l}}{2^{l}-1} A_{l} \nu_{0}+(2 i)^{-p} M_{p} \nu_{0}+\nu_{p} \\
= & (2 i)^{-p}\left\{\sum_{l=1}^{p} \frac{\left(\begin{array}{l}
p \\
l
\end{array}\right)}{2^{l}-1} M_{p-l} A_{l}+M_{p}\right\} \nu_{0}+\nu_{p}=(2 i)^{-p} A_{p} \nu_{0}+\nu_{p} .
\end{aligned}
$$

Thus, equations (2.12)-(2.14) are equivalent to

$$
B_{n} \nu_{0}=0, \nu_{n}=\frac{(2 i)^{-n}}{2^{n}-1} A_{n} \nu_{0}, n=1,2, \ldots, p .
$$

So we have proved this theorem.

\section{Computation of the derivatives $G^{(j)}(1)$}

Theorem 2.3 propose a sufficient and necessary condition for the corresponding scaling functions to have accuracy of degree $p$. Note that this implies the necessity of computing the derivatives of $G(x)$, that is, the derivatives of the product of several functions. Next, we give the following results concerning the computation of derivatives.

\subsection{Derivation of products of functions}

The first lemma is classical in mathematical analysis which is called Leibniz's formula.

Lemma 3.1. Let $f(x)=f_{1}(x) f_{2}(x)$. Then, for any natural number $n$, the $n$-th derivative of function $f$ is

$$
f^{(n)}(x)=\sum_{m=0}^{n}\left(\begin{array}{l}
n \\
m
\end{array}\right) f_{1}^{(m)}(x) f_{2}^{(n-m)}(x) .
$$

Lemma 3.2. Let $f(x)=f_{1}(x) \cdots f_{M}(x)$. Then, for any natural number $n$, the $n$-th derivative of $f$ is

$$
\left(f_{1} \cdots f_{M}\right)^{(n)}(x)=\sum_{\substack{j_{1}+\cdots+j_{M}=n \\ j_{i} \geq 0}} \frac{n !}{j_{1} ! j_{2} ! \cdots j_{M} !} f_{1}^{\left(j_{1}\right)}(x) \cdots f_{M}^{\left(j_{M}\right)}(x) .
$$

Proof. We will prove this theorem by induction of $M$. For $M=1$, this theorem holds naturally. And, Lemma 3.1 states that (3.2) holds for $M=2$. 
Suppose this theorem holds for some natural number $M \geq 2$, then, by Lemma 3.1,

$$
\begin{aligned}
& \left(f_{1} \cdots f_{M+1}\right)^{(n)}(x) \\
= & \sum_{m=0}^{n}\left(\begin{array}{c}
n \\
m
\end{array}\right)\left(f_{1} \cdots f_{M}\right)^{(m)}(x) f_{M+1}^{(n-m)}(x) \\
= & \sum_{m=0}^{n}\left(\begin{array}{c}
n \\
m
\end{array}\right) \sum_{\substack{j_{1}+\cdots+j_{M}=m \\
j_{i} \geq 0}} \frac{m !}{j_{1} ! j_{2} ! \cdots j_{M} !} f_{1}^{\left(j_{1}\right)}(x) \cdots f_{M}^{\left(j_{M}\right)}(x) f_{M+1}^{(n-m)}(x) \\
= & \sum_{j_{1}+\cdots+j_{M+1}=n} \frac{(n+1) !}{j_{1} ! j_{2} ! \cdots j_{M+1} !} f_{1}^{\left(j_{1}\right)}(x) \cdots f_{M+1}^{\left(j_{M+1}\right)}(x) .
\end{aligned}
$$

Thus, this theorem also holds for $M+1$.

\subsection{Computation of the derivatives $G^{(j)}(1)$}

In this section we will concentrate on the filter banks which are constructed in (1.5)-(1.7). Let $f_{0}=\frac{1}{2}\left(I_{r}, x I_{r}\right)$, and for $j=1, \ldots, N$,

$$
f_{j}(x)=U_{j} \operatorname{diag}\left(I_{r}, x^{2} I_{r}\right) U_{j}^{T} .
$$

Then their derivatives are

$$
f_{0}^{(k)}(1)=\left\{\begin{array}{ll}
\frac{1}{2}\left(I_{r}, I_{r}\right), & k=0, \\
\frac{1}{2}\left(0_{r}, I_{r}\right), & k=1, \\
0_{r \times 2 r}, & k \geq 2,
\end{array} \quad f_{j}^{(k)}(1)= \begin{cases}I_{2 r}, & k=0, \\
2 U_{j} \operatorname{diag}\left(0_{r}, I_{r}\right) U_{j}^{T}, & k=1,2, \\
0_{2 r}, & k \geq 3 .\end{cases}\right.
$$

Or equivalently,

$$
\begin{aligned}
f_{0}^{(k)}(1) & =\frac{1}{2}\left(\delta_{k} I_{r},\left(\delta_{k}+\delta_{k-1}\right) I_{r}\right), \\
f_{j}^{(k)}(1) & =U_{j} \operatorname{diag}\left(\delta_{k} I_{r},\left(\delta_{k}+2 \delta_{k-1}+2 \delta_{k-2}\right) I_{r}\right) U_{j}^{T} .
\end{aligned}
$$

Thus, by Lemma 3.2 , the derivative of $G$ at $x=1$ is given as in the next theorem.

Theorem 3.3. The derivatives of $G(x)$ is given as

$$
\begin{aligned}
& G^{(k)}(1) \\
= & \frac{1}{2}\left(I_{r}, I_{r}\right) \sum_{\substack{k_{1}+\cdots+k_{N}=k, 0 \leq k_{i} \leq 2}} \frac{k !}{k_{1} ! \cdots k_{M} !} \prod_{j=1}^{N} U_{j} \operatorname{diag}\left(\delta_{k_{j}} 0_{r},\left(\delta_{k_{j}}+2 \delta_{k_{j}-1}+2 \delta_{k_{j}-2}\right) I_{r}\right) U_{j}^{T} \\
& +\frac{1}{2}\left(0_{r}, I_{r}\right) \sum_{\substack{k_{1}+\cdots+k_{N}=k-1 \\
0 \leq k_{i} \leq 2}} \frac{(k-1) !}{k_{1} ! \cdots k_{M} !} \prod_{j=1}^{N} U_{j} \operatorname{diag}\left(\delta_{k_{j}} 0_{r},\left(\delta_{k_{j}}+2 \delta_{k_{j}-1}+2 \delta_{k_{j}-2}\right) I_{r}\right) U_{j}^{T} .
\end{aligned}
$$


Proof. By using Lemma 3.2 and relation (3.3), we have

$$
\begin{aligned}
G^{(k)}(1)= & \frac{1}{2}\left(I_{r}, I_{r}\right) \sum_{\substack{k_{1}+\cdots+k_{N}=k \\
0 \leq k_{i} \leq 2}} \frac{k !}{k_{1} ! \cdots k_{M} !} f_{1}^{\left(k_{1}\right)}(1) \cdots f_{N}^{\left(k_{N}\right)}(1) \\
& +\frac{1}{2}\left(0_{r}, I_{r}\right) \sum_{\substack{k_{1}+\cdots+k_{N}=k-1 \\
0 \leq k_{i} \leq 2}} \frac{(k-1) !}{k_{1} ! \cdots k_{M} !} f_{1}^{\left(k_{1}\right)}(1) \cdots f_{N}^{\left(k_{N}\right)}(1) .
\end{aligned}
$$

This combined with relations (3.4)-(3.5) concludes the proof of this theorem.

Although this theorem gives a close form of the derivative $G^{(j)}(1)$, it is not very convenient for computational purpose. It is implied from relations (3.3) and (3.6) that to compute $G^{(k)}(1)$, we should consider matrix multiplication of the following form $\prod_{q=1}^{M} U_{j_{q}} \operatorname{diag}\left(0_{r}, I_{r}\right) U_{j_{q}}{ }^{T}$ where each $U_{j_{q}}$ is characterized as in (1.7).

Proposition 3.4. Let $U_{j}$ be characterized as in (1.7). Then for any $2 \leq M \leq$ $N$, we have

$$
\prod_{j=1}^{M} U_{j}\left(\begin{array}{ll}
{ }^{0_{r}} & \\
& I_{r}
\end{array}\right) U_{j}^{T}=U_{1}\left(\begin{array}{cc}
0_{r} & 0_{r} \\
0_{r} & \prod_{j=1}^{M-1}\left(Q_{j}^{T} Q_{j+1}+J_{r} P_{j}^{T} P_{j+1} J_{r}\right)
\end{array}\right) U_{M}^{T} .
$$

Proof. We will prove this proposition by induction of $M$.

(1) For $M=2$, by using the fact that

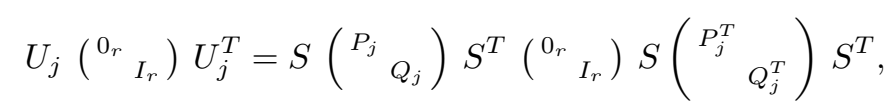

we have

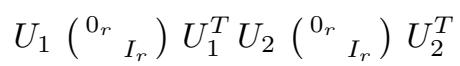

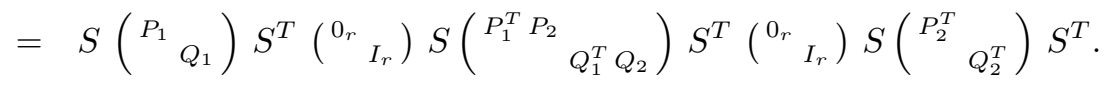

Note that for any $r \times r$ matrices $A, B, C, D$, the following identities hold:

$$
\begin{aligned}
& \left(\begin{array}{cc}
0_{r} & \\
& I_{r}
\end{array}\right)\left(\begin{array}{ll}
A & B \\
C & D
\end{array}\right)\left(\begin{array}{cc}
0_{r} & \\
& I_{r}
\end{array}\right)=\left(\begin{array}{cc}
0_{r} & 0_{r} \\
0_{r} & D
\end{array}\right), \\
& S\left(\begin{array}{cc}
P_{1}^{T} P_{2} & \\
& Q_{1}^{T} Q_{2}
\end{array}\right) S^{T}=\left(\begin{array}{cc}
* & * \\
* & Q_{1}^{T} Q_{2}+J_{r} P_{1}^{T} P_{2} J_{r}
\end{array}\right)
\end{aligned}
$$

Thus, we have

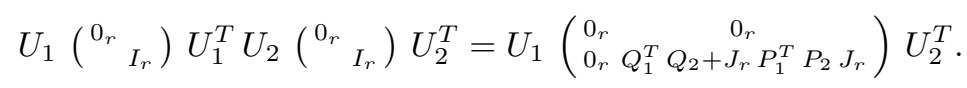

That is, we have proved the relation (3.7) for $M=2$. 
(2) Suppose relation (3.7) holds for some $M \geq 2$, then, by induction,

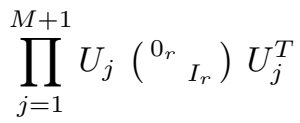

$$
\begin{aligned}
& =U_{1}\left(\begin{array}{c}
0_{r} \\
0_{r} \prod_{j=1}^{M-1}\left(Q_{j}^{T} Q_{j+1}{ }^{0_{r}}+J_{r} P_{j}^{T} P_{j+1} J_{r}\right)
\end{array}\right) U_{M}^{T} U_{M+1}\left(\begin{array}{cc}
0_{r} & \\
& I_{r}
\end{array}\right) U_{M+1}^{T} .
\end{aligned}
$$

Note that for any $r \times r$ matrices $A, B, C, D, F$ the following identities hold:

$$
\begin{aligned}
& \left(\begin{array}{cc}
0_{r} & 0_{r} \\
0_{r} & F
\end{array}\right)\left(\begin{array}{cc}
A & B \\
C & D
\end{array}\right)\left(\begin{array}{cc}
0_{r} & \\
& I_{r}
\end{array}\right)=\left(\begin{array}{cc}
0_{r} & 0_{r} \\
0_{r} & F D
\end{array}\right) \\
& U_{M}^{T} U_{M+1}=S\left(\begin{array}{cc}
P_{M}^{T} P_{M+1} & \\
& Q_{M}^{T} Q_{M+1}
\end{array}\right) S^{T} \\
& =\left(\begin{array}{cc}
* & * \\
* & Q_{M}^{T} Q_{M+1}+J_{r} P_{M}^{T} P_{M+1} J_{r}
\end{array}\right) .
\end{aligned}
$$

Consequently,

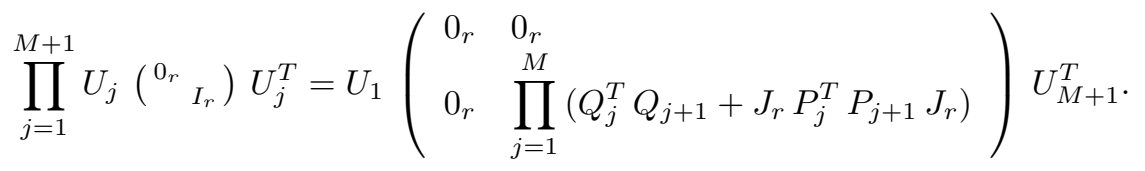

That is, we have proved that this proposition holds for $M+1$.

Another question concerning the formula (3.6) is as follows: how to characterize the set $\left\{\left(j_{1}, \ldots, j_{N}\right): j_{1}+\cdots+j_{N}=k, j_{i} \in\{0,1,2\}\right\}$. In fact we only have to consider the following simpler sets

$$
S_{N, k}:=\left\{\left(j_{1}, \ldots, j_{N}\right): j_{1} \geq j_{2} \geq \cdots \geq j_{N}, j_{1}+\cdots+j_{N}=k, j_{i} \in\{0,1,2\}\right\} .
$$

Proposition 3.5. Given a natural number $N$, suppose $S_{N, k}$ are defined in (3.12) for all nonnegative integers $k$. Then, we have

$$
S_{N, k}= \begin{cases}\{(0,0, \ldots, 0)\}, & k=0, \\ \{(1,0, \ldots, 0)\}, & k=1, \\ \{(\overbrace{1, \ldots, 1}^{k}, 0, \ldots, 0)\} \cup \tilde{S}_{N, k-2}, & 2 \leq k \leq N, \\ \left\{\left(2-j_{N}, \ldots, 2-j_{1}\right):\left(j_{1}, \ldots, j_{N}\right) \in S_{N, 2 N-k}\right\}, & N+1 \leq k \leq 2 N, \\ \varnothing, & k \geq 2 N+1,\end{cases}
$$

with $\tilde{S}_{N, k}:=\left\{\left(2, j_{1}, \ldots, j_{N-1}\right):\left(j_{1}, \ldots, j_{N}\right) \in S_{N, k}\right\}$. Moreover, the cardinalities are

$$
\#\left(S_{N, k}\right)= \begin{cases}{\left[\frac{k}{2}\right]+1} & 0 \leq k \leq N \\ N-\left[\frac{k-1}{2}\right] & N+1 \leq k \leq 2 N \\ 0 & k \geq 2 N+1\end{cases}
$$


Proof. To verify the equalities (3.13), we only have to prove that the third equality holds for all $2 \leq k \leq N$, that is, $S_{N, k}=\{(\overbrace{1, \ldots, 1}^{k}, 0, \ldots, 0)\} \cup$ $\tilde{S}_{N, k-2}$. On the one hand, for any $\left(j_{1}, \ldots, j_{N}\right) \in S_{N, k}$, we have either $j_{1}=2$ or $j_{1}=1$. If $j_{1}=2$, then it is easy to verify that $\left(j_{2}, \ldots, j_{N}, 0\right) \in S_{N, k-2}$, thus, $\left(j_{1}, \ldots, j_{N}\right) \in \tilde{S}_{N, k-2}$; in the case that $j_{1}=1$, we have $\left(j_{1}, \ldots, j_{N}\right)=$ $(\overbrace{1, \ldots, 1}^{k}, 0, \ldots, 0)$. So we have prove that

$$
S_{N, k} \subseteq\{(\overbrace{1, \ldots, 1}^{k}, 0, \ldots, 0)\} \bigcup \tilde{S}_{N, k-2} .
$$

On the other hand, firstly we know that $\overbrace{1, \ldots, 1}^{k}, 0, \ldots, 0) \in S_{N, k}$. For any $\left(2, j_{1}, \ldots, j_{N-1}\right) \in \tilde{S}_{N, k-2}$ where $\left(j_{1}, \ldots, j_{N}\right) \in S_{N, k-2}$, one can check easily that $j_{N}=0$. Otherwise, if $j_{N} \geq 1$, then it is implied that $j_{1}+\cdots+j_{N} \geq$ $N>k-2$. Thus, we have $\left(2, j_{1}, \ldots, j_{N-1}\right) \in S_{N, k}$. Consequently, we have proved that $S_{N, k} \supseteq\{(\overbrace{1, \ldots, 1}^{k}, 0, \ldots, 0)\} \cup \tilde{S}_{N, k-2}$. Combining the two result, the proof of (3.13) is finished. The equality (3.14) can be easily checked by using the results of (3.13).

Proposition 3.5 produces a recursive method to construct the sets $S_{N, k}$. In what follows we will show exactly what $S_{N, k}$ are.

Proposition 3.6. Let the sets $S_{N, k}$ be defined as in (3.12). Then for any $0 \leq k \leq N$, we have

$$
S_{N, k}=\bigcup_{j=0}^{\left[\frac{k}{2}\right]}\{(\overbrace{2, \ldots, 2}^{j}, \overbrace{1, \ldots, 1}^{k-2 j}, \overbrace{0, \ldots, 0}^{N-k+j})\} .
$$

And, for those $N+1 \leq k \leq 2 N$, we have

$$
\left.S_{N, k}=\bigcup_{j=0}^{\left[N-\frac{k}{2}\right]}\{\overbrace{2, \ldots, 2}^{k+j-N}, \overbrace{1, \ldots, 1}^{2 N-k-2 j}, \overbrace{0, \ldots, 0}^{j})\right\} .
$$

Proof. One can easily checks that equality (3.16) is implied by (3.13) and (3.15). We will prove by induction of $k$ that equality (3.15) holds for all $0 \leq k \leq N$. Firstly, it is easy to verify that this holds for $k=0,1$. Furthermore, the fact that $S_{N, 2}=\{(2,0,0, \ldots, 0),(1,1,0, \ldots, 0)\}$ implies that this equality also holds for $k=2$.

Suppose there are some $2 \leq k \leq N-1$, such that equality (3.15) holds for all $0 \leq n \leq k$. By (3.13), we have

$$
\begin{aligned}
S_{N, k+1} & =\{(\overbrace{1, \ldots, 1}^{k+1}, 0, \ldots, 0)\} \cup \tilde{S}_{N, k-1}, \\
\text { with } \quad \tilde{S}_{N, k-1} & =\left\{\left(2, j_{1}, \ldots, j_{N-1}\right):\left(j_{1}, \ldots, j_{N}\right) \in S_{N, k-1}\right\} .
\end{aligned}
$$


As was pointed in the proof of the last proposition, for any $\left(j_{1}, \ldots, j_{N}\right) \in$ $S_{N, k-1}$, we have $j_{N}=0$. Thus, by induction,

$$
\tilde{S}_{N, k-1}=\bigcup_{j=0}^{\left[\frac{k-1}{2}\right]}\{(\overbrace{2, \ldots, 2}^{j+1}, \overbrace{1, \ldots, 1}^{k-1-2 j}, \overbrace{0, \ldots, 0}^{N-k+j}\},
$$

so that

$$
\begin{aligned}
& S_{N, k+1}=\{(\overbrace{1, \ldots, 1}^{k+1}, 0, \ldots, 0)\} \cup \bigcup_{j=0}^{\left[\frac{k-1}{2}\right]}\{(\overbrace{2, \ldots, 2}^{j+1}, \overbrace{1, \ldots, 1}^{k-1-2 j}, \overbrace{0, \ldots, 0}^{N-k+j}\}, \\
& (3.18)=\bigcup_{j=0}^{\left[\frac{k+1}{2}\right]}\{(\overbrace{2, \ldots, 2}^{j}, \overbrace{1, \ldots, 1}^{k+1-2 j}, \overbrace{0, \ldots, 0}^{N-k-1+j})\} .
\end{aligned}
$$

Note that to prove the equality (3.18), we have used the equality $\left[\frac{k+1}{2}\right]-1=$ $\left[\frac{k-1}{2}\right]$.

Combining the results in relation (3.6), and Propositions 3.4, 3.6, we propose the following theorem which seems more convenient to compute $G^{(j)}(1)$.

Theorem 3.7. For $0 \leq k \leq N$, we have

$$
\begin{aligned}
& \sum_{\substack{k_{1}+\cdots+k_{N}=k \\
0 \leq k_{i} \leq 2}} \frac{k !}{k_{1} ! \cdots k_{M} !} f_{1}^{\left(k_{1}\right)}(1) \cdots f_{N}^{\left(k_{N}\right)}(1) \\
& =\sum_{j=0}^{\left[\frac{k}{2}\right]}\left(\begin{array}{c}
k-j \\
j
\end{array}\right) \sum_{\left(1 \leq p_{1}<\cdots<p_{k-j} \leq N\right)} 2^{k-2 j} k ! U_{p_{1}}\left(\begin{array}{cc}
0_{r} & 0_{r} \\
& \prod_{l=1}^{k-j-1} \\
0_{r} & \left(Q_{p_{l}}^{T} Q_{p_{l+1}}+J P_{p_{l}}^{T} P_{p_{l+1}} J\right)
\end{array}\right) U_{p_{k-j}}^{T} .
\end{aligned}
$$

And, for $N+1 \leq k \leq 2 N$, we have

$$
\begin{aligned}
& \sum_{\substack{k_{1}+\cdots+k_{N}=k \\
0 \leq k_{i} \leq 2}} \frac{k !}{k_{1} ! \cdots k_{M} !} f_{1}^{\left(k_{1}\right)}(1) \cdots f_{N}^{\left(k_{N}\right)}(1) \\
= & \sum_{j=0}^{\left[N-\frac{k}{2}\right]}\left(\begin{array}{c}
N-j \\
k+j-N
\end{array}\right) \sum_{\substack{\left.1 \leq p_{1}<\cdots<p_{N-j} \leq N\right) \\
2^{k+j-N}}} U_{p_{1}}\left(\begin{array}{cc}
0_{r} & 0_{r} \\
0_{r} & \prod_{l=1}^{N-j-1}\left(Q_{p_{l}}^{T} Q_{p_{l}+1}+J P_{p_{l}}^{T} P_{p_{l}+1} J\right)
\end{array}\right) U_{p_{N-j}^{T}}^{T} .
\end{aligned}
$$

Proof. For any $n$-tuples $\left(a_{1}, \ldots, a_{n}\right)$, denotes by $P\left(a_{1}, \ldots, a_{n}\right)$ the set of permutations of $a_{1}, \ldots, a_{n}$. First consider the case $0 \leq k \leq N$, we have

$$
\begin{aligned}
& \sum_{\substack{k_{1}+\cdots+k_{N}=k \\
0 \leq k_{i} \leq 2}} \frac{k !}{k_{1} ! \cdots k_{M} !} f_{1}^{\left(k_{1}\right)}(1) \cdots f_{N}^{\left(k_{N}\right)}(1) \\
= & \sum_{\left(j_{1}, \ldots, j_{N}\right) \in S_{N, k}\left(k_{1}, \ldots, k_{N}\right) \in P\left(j_{1}, \ldots, j_{N}\right)} \frac{k !}{k_{1} ! \cdots k_{M} !} f_{1}^{\left(k_{1}\right)}(1) \cdots f_{N}^{\left(k_{N}\right)}(1)
\end{aligned}
$$




$$
\begin{aligned}
& =\sum_{\left(j_{1}, \ldots, j_{N}\right) \in S_{N, k}} \sum_{\left(k_{1}, \ldots, k_{N}\right) \in P\left(j_{1}, \ldots, j_{N}\right)} \frac{k !}{j_{1} ! \cdots j_{M} !} f_{1}^{\left(k_{1}\right)}(1) \cdots f_{N}^{\left(k_{N}\right)}(1) \\
& =\sum_{j=0}^{\left[\frac{k}{2}\right]}\left[\sum_{\left(k_{1}, \ldots, k_{N}\right) \in P\left(e_{j, k}\right)} \frac{k !}{2^{j}} f_{1}^{\left(k_{1}\right)}(1) \cdots f_{N}^{\left(k_{N}\right)}(1)\right]
\end{aligned}
$$

where the $N$-tuples $e_{j, k}$ are defined as $e_{j, k}:=(\overbrace{2, \ldots, 2}^{j}, \overbrace{1, \ldots, 1}^{k-2 j}, \overbrace{0, \ldots, 0})$, note that the last equality holds due to Proposition 3.6. Next, we will show clearly what the summation in the bracket is. For any given $j=0,1, \ldots,\left[\frac{k}{2}\right]$, let

$$
\begin{gathered}
C_{1}:=\sum_{\left(k_{1}, \ldots, k_{N}\right) \in P\left(e_{j, k}\right)} \frac{k !}{2^{j}} f_{1}^{\left(k_{1}\right)}(1) \cdots f_{N}^{\left(k_{N}\right)}(1), \\
C_{2}:=\sum_{\left(q_{1}, \ldots, q_{k-j}\right) \in p\left(\tilde{e}_{j, k}\right)} \sum_{1 \leq p_{1}<\cdots<p_{k-j} \leq N} \frac{k !}{2^{j}} f_{p_{1}}^{\left(q_{1}\right)}(1) \cdots f_{p_{k-j}}^{\left(q_{k-j}\right)}(1),
\end{gathered}
$$

where $\tilde{e}_{j, k}:=(\overbrace{2, \ldots, 2}^{j}, \overbrace{1, \ldots, 1}^{k-2 j})$, we claim that $C_{1}=C_{2}$. On the one hand, it is straightforward to prove that the terms of summations are equal since $\left(\begin{array}{c}N \\ j\end{array}\right)\left(\begin{array}{c}N-j \\ k-2 j\end{array}\right)=\left(\begin{array}{c}k-j \\ j\end{array}\right)\left(\begin{array}{c}N \\ k-j\end{array}\right)$. On the other hand, we will verify that any summation term of $C_{1}$ emerges also in $C_{2}$. For fixed permutation of $e_{j, k}$, it is implied from relation (3.3) that only $k-j$ terms in the product $\frac{k !}{2^{j}} f_{1}^{\left(k_{1}\right)}(1) \cdots f_{N}^{\left(k_{N}\right)}(1)$ counts. Thus, there exists $1 \leq p_{1}<\cdots<p_{k-j} \leq N$, and $q_{1}, \ldots, q_{k-j}$ being permutation of $\tilde{e}_{j, k}$ so that $\frac{k !}{2^{j}} f_{1}^{\left(k_{1}\right)}(1) \cdots f_{N}^{\left(k_{N}\right)}(1)=\frac{k !}{2^{j}} f_{p_{1}}^{\left(q_{1}\right)}(1) \cdots f_{p_{k-j}}^{\left(q_{k-j}\right)}(1)$. This concludes the proof of identity $C_{1}=C_{2}$. So we have

$$
\begin{aligned}
& \sum_{\substack{k_{1}+\cdots+k_{N}=k \\
0 \leq k_{i} \leq 2}} \frac{k !}{k_{1} ! \cdots k_{M} !} f_{1}^{\left(k_{1}\right)}(1) \cdots f_{N}^{\left(k_{N}\right)}(1) \\
& =\sum_{j=0}^{\left[\frac{k}{2}\right]}\left[\sum_{\left(q_{1}, \ldots, q_{k-j}\right) \in P\left(\tilde{e}_{j, k}\right)} \sum_{1 \leq p_{1}<\cdots<p_{k-j} \leq N} \frac{k !}{2^{j}} f_{p_{1}}^{\left(q_{1}\right)}(1) \cdots f_{p_{k-j}}^{\left(q_{k-j}\right)}(1)\right] \\
& =\sum_{j=0}^{\left[\frac{k}{2}\right]}\left(\begin{array}{c}
k-j \\
j
\end{array}\right) \sum_{\left(1 \leq p_{1}<p_{2}<\cdots<p_{k-j} \leq N\right)} \frac{k !}{2^{j}} f_{p_{1}}^{\left(q_{1}\right)}(1) \cdots f_{p_{k-j}}^{\left(q_{k-j}\right)}(1) \\
& =\sum_{j=0}^{\left[\frac{k}{2}\right]}\left(\begin{array}{c}
k-j \\
j
\end{array}\right) \sum_{\left(1 \leq p_{1}<p_{2}<\cdots<p_{k-j} \leq N\right)} 2^{2^{k-2 j} k ! U_{p_{1}}}\left(\begin{array}{cc}
0_{r} & 0_{r} \\
0_{r} & \prod_{l=1}^{k-j-1}\left(Q_{p_{l}}^{T} Q_{1+p_{l}}+J P_{p_{l}}^{T} P_{1+p_{l}} J\right)
\end{array}\right) U_{p_{k-j}}^{T} .
\end{aligned}
$$


It should be noted that we have used Proposition 3.4 and the following facts:

(1) for any $j=1, \ldots, N, f_{j}^{(1)}(1)=f_{j}^{(2)}(1)=2 U_{j}\left(\begin{array}{cc}0_{r} & \\ & I_{r}\end{array}\right) U_{j}^{T}$;

(2) the number of permutations of $(\overbrace{2, \ldots, 2}^{m}, \overbrace{1, \ldots, 1}^{n})$ is $\left(\begin{array}{c}m+n \\ m\end{array}\right)$.

By the same trick we can prove the theorem for the case $N+1 \leq k \leq 2 N$.

\section{Numerical examples}

Consider the case $r=2$. Assume that filter banks are constructed as in (1.5)-(1.7) where the $2 \times 2$ real orthogonal matrices $P_{j}, Q_{j}$ are

$$
P_{j}=\left(\begin{array}{cc}
\cos \alpha_{j} & \sin \alpha_{j} \\
-\sin \alpha_{j} & \cos \alpha_{j}
\end{array}\right), Q_{j}=\left(\begin{array}{cc}
\cos \beta_{j} & \sin \beta_{j} \\
-\sin \beta_{j} & \cos \beta_{j}
\end{array}\right) .
$$

Let $\gamma_{j}=\alpha_{j}+\beta_{j}$, then the matrices $B_{1}, B_{2}$ defined in Theorem 2.3 are

$$
B_{1}=-\lambda_{3} I_{2}, B_{2}=\left(\begin{array}{cc}
\lambda_{2} & 0 \\
0 & \lambda_{3}
\end{array}\right) \text {, }
$$

with parameters

$$
\begin{aligned}
& \lambda_{1}=\frac{1}{2}+\sum_{j=1}^{N} \cos \gamma_{j}, \\
& \lambda_{2}=-N+\sum_{j=1}^{N} \sin \gamma_{j}-(N+1) \sum_{j=1}^{N} \cos \gamma_{j}+\sum_{1 \leq k<j \leq N} \sin \left(\gamma_{j}-\gamma_{k}\right), \\
& \lambda_{3}=-N-\sum_{j=1}^{N} \sin \gamma_{j}-(N+1) \sum_{j=1}^{N} \cos \gamma_{j}-\sum_{1 \leq k<j \leq N} \sin \left(\gamma_{j}-\gamma_{k}\right) .
\end{aligned}
$$

Thus, by Theorem 2.3, we have the following sufficient and necessary conditions for the corresponding scaling functions to have accuracy $p=2,3$.

Theorem 4.1. When $r=2$, and wavelet filter banks are constructed as in (1.5)-(1.7) and (4.19), then the corresponding scaling functions have at least accuracy of order $p=2$ if and only if

$$
\sum_{j=1}^{N} \cos \gamma_{j}=-\frac{1}{2}
$$

Moreover, the corresponding scaling functions have at least accuracy of order $p=3$ if and only if in addition to (4.20), the following equality holds:

$$
\sum_{j=1}^{N} \sin \gamma_{j}+\sum_{1 \leq k<j \leq N} \sin \left(\gamma_{j}-\gamma_{k}\right)= \pm \frac{1}{2}
$$


Proof. By Theorem 2.3, the scaling functions have at least second accuracy if and only if there exists nonzero $2 \times 1$ vector $\nu_{0}$ such that $B_{1} \nu_{0}=-\lambda_{1} \nu_{0}=0$, this reduces to $\lambda_{1}=0$.

Similarly, the scaling functions have at least third accuracy if and only if there exists nonzero $2 \times 1$ vector $\nu_{0}$ such that $B_{1} \nu_{0}=-\lambda_{1} \nu_{0}=0, B_{2} \nu_{0}=$ $\left(\begin{array}{cc}\lambda_{2} & 0 \\ 0 & \lambda_{3}\end{array}\right) \nu_{0}=0$, this reduces to either of the following two equalities:

$$
\left\{\begin{array} { l } 
{ \lambda _ { 1 } = 0 , } \\
{ \lambda _ { 2 } = 0 , }
\end{array} \quad \left\{\begin{array}{l}
\lambda_{1}=0, \\
\lambda_{3}=0 .
\end{array}\right.\right.
$$
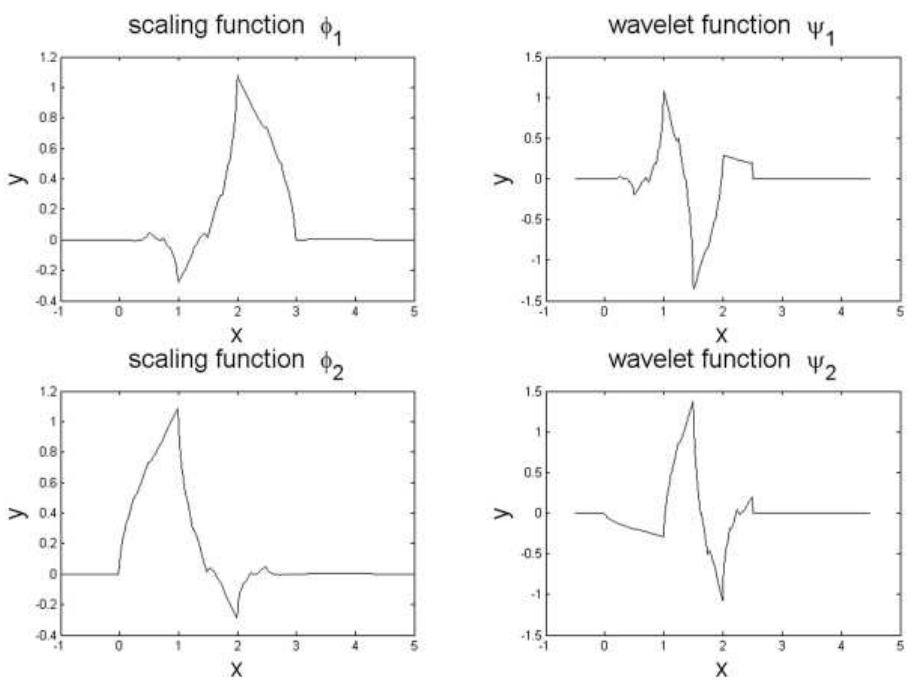

FIGURE 1. Wavelet and scaling function with second accuracy

By equation (4.20), to obtain second accuracy and the minimal length of the filters, we should choose $N=1$, that is, $\cos \gamma_{1}=-\frac{1}{2}$. In fact, in this case, $\psi_{2}, \phi_{2}$ are the Daubechies's wavelet function db2 and the corresponding scaling function. The graph of the above functions are plotted in Figure 1 .

On the other hand, to obtain third accuracy and minimal length, we have to choose $N=2$. Here the equations (4.20) and (4.21) have four solutions:

$$
\begin{aligned}
& \gamma_{1}=\pi+\arcsin 0.5374, \quad \gamma_{2}=-\arcsin 0.9392 \\
& \gamma_{1}=\pi+\arcsin 0.9756, \quad \gamma_{2}=\pi-\arcsin 0.9600 \\
& \gamma_{1}=\pi-\arcsin 0.5374, \quad \gamma_{2}=-\arcsin 0.9392 ; \\
& \gamma_{1}=\pi-\arcsin 0.9756, \quad \gamma_{2}=\pi+\arcsin 0.9600 .
\end{aligned}
$$

Take the first solution, and we present the graph of the above functions in Figure 2. 

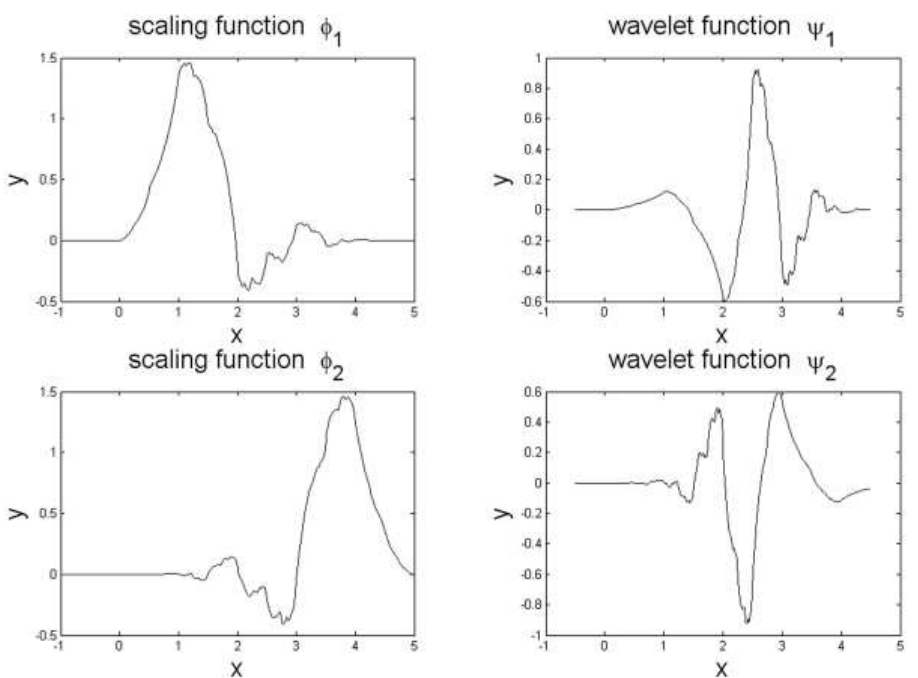

FiguRE 2. Wavelet and scaling function with third accuracy

\section{References}

[1] C. Cabrelli, C. Heil, and U. Molter, Accuracy of lattice translates of several multidimensional refinable functions, J. Approx. Theory 95 (1998), no. 1, 5-52.

[2] Q. H. Chen, C. A. Micchelli, S. Peng, and Y. Xu, Multivariate filter banks having matrix factorizations, SIAM J. Matrix Anal. Appl. 25 (2003), no. 2, 517-531.

[3] I. Daubechies, Ten Lectures on Wavelets, CBMS-NSF Regional Conference Series in Applied Mathematics, 61. Society for Industrial and Applied Mathematics (SIAM), Philadelphia, PA, 1992.

[4] C. deBoor, R. A. Devore, and A. Ron, Approximation orders of FSI spaces in $L_{2}\left(\mathbf{R}^{d}\right)$, Constr. Approx. 14 (1998), no. 4, 631-652.

[5] C. Heil, G. Strang, and V. Strela, Approximation by translates of refinable functions, Numer. Math. 73 (1996), no. 1, 75-94.

[6] R. Q. Jia, Approximation properties of multivariate wavelets, Math. Comp. 67 (1998), no. $222,647-665$

[7] Q. Lian, H. Xiao, and Q. Chen, Some properties on multivariate filter banks with a matrix factorization, Progr. Natur. Sci. (English Ed.) 15 (2005), no. 2, 115-125.

[8] G. Plonka, Approximation order provided by refinable function vectors, Constr. Approx. 13 (1997), no. 2, 221-244

[9] H. Xiao, Lattice structure for paraunitary linear-phase filter banks with accuracy, Acta Math. Sin. (Engl. Ser.) 22 (2006), no. 3, 679-688.

[10] _ Piecewise Linear Spectral Sequences and Wavelet Filter Banks, Doctoral Thesis, Graducate School of CAS, May 2005.

College of SCIENCE

China Three Gorges University

Yichang 443002, P. R. CHinA

E-mail address: hongying_x@ctgu.edu.cn 